Artículos Científicos

\title{
Estudio evaluativo del Sistema Institucional de Tutorías para el seguimiento de trayectorias escolares
}

\begin{abstract}
Assessment Study of the Institutional Tutoring System for Monitoring School
Trajectories
\end{abstract}

Estudo de avaliação do Sistema de Tutoria Institucional do acompanhamento de trajetórias escolares

Fernando E. Sánchez Martínez

Universidad Autónoma del Carmen, México

fsanchez@pampano.unacar.mx https://orcid.org/0000-0003-4458-5402

Ricardo A. Barrera Cámara Universidad Autónoma del Carmen, México rbarrera@pampano.unacar.mx https://orcid.org/0000-0002-3170-4671

Ana Canepa Sáenz Universidad Autónoma del Carmen, México acanepa@pampano.unacar.mx https://orcid.org/0000-0003-0583-439X 


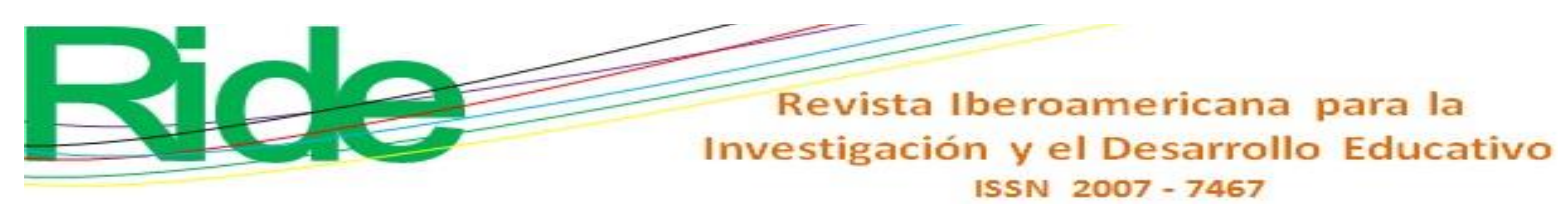

\title{
Resumen
}

El propósito del presente estudio fue evaluar y reunir información sobre la experiencia y necesidades de los profesores que participan en el Programa Institucional de Tutoría (PIT) y que dan seguimiento y apoyo en las trayectorias escolares de estudiantes de nivel superior. La investigación fue de corte cuantitativo con un alcance descriptivo. El instrumento utilizado fue una encuesta en línea que se aplicó a 114 tutores que están adscritos a las diversas dependencias de educación superior de la Universidad Autónoma del Carmen, donde 43.86 \% tiene una experiencia superior a los 10 años como tutor. Entre los hallazgos destaca que el Sistema Institucional de Tutorías (SIT) no cuenta con los indicadores suficientes para realizar un seguimiento de trayectoria escolar eficiente y que $42.11 \%$ de los tutores realiza manualmente un seguimiento individual de acuerdo con su experiencia y necesidades de información. A partir de este estudio se identificaron los indicadores de trayectoria escolar que requieren los tutores para dar un seguimiento exitoso.

Palabras clave: control de rendimiento escolar, deserción escolar, fracaso escolar, indicadores educativos, rezago escolar, seguimiento escolar, trayectoria académica, tutoría.

\begin{abstract}
The purpose of the present study was to assess and gather information about the experience and needs of the teachers who participate in the Institutional Tutorial Program (PIT), giving follow-up and support in the school trajectories of higher level students. The investigation was of a quantitative nature with a descriptive scope. The instrument used was an online survey that was applied to 114 tutors who are attached to the various higher education units of the Universidad Autónoma del Carmen, where $43.86 \%$ have more than 10 years of experience as a tutor. Among the findings, the Institutional Tutoring System (SIT) does not have enough indicators to monitor the efficient school trajectory and $42.11 \%$ of the tutors manually follow up individually according to their experience and information needs. From this study, indicators of school trajectories that tutors require to follow up successfully were identified.
\end{abstract}

Keywords: control of school performance, school dropout, school failure, educational indicators, school lag, school monitoring, academic trajectory, tutoring. 


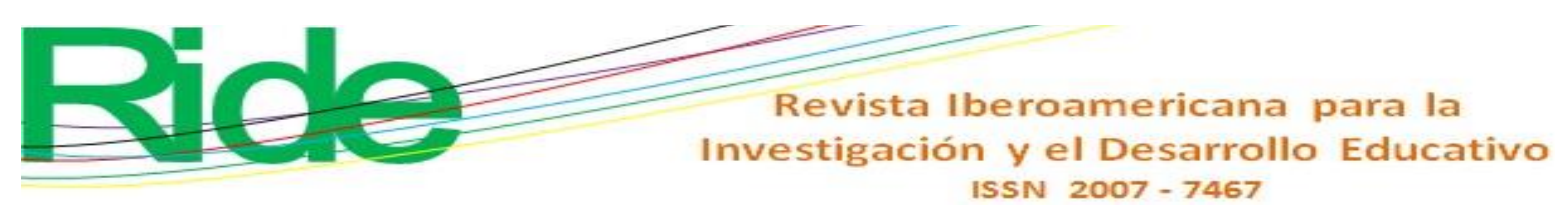

\section{Resumo}

O objetivo do presente estudo foi avaliar e coletar informações sobre a experiência e as necessidades dos professores que participam do Programa de Tutoria Institucional (PIT) e que fornecem acompanhamento e apoio nas trajetórias escolares de alunos de nível superior. A investigação foi quantitativa, com escopo descritivo. $O$ instrumento utilizado foi uma pesquisa online aplicada a 114 tutores vinculados às diversas unidades de ensino superior da Universidade Autônoma de Carmen, onde 43,86\% têm experiência de mais de 10 anos como tutor. Entre os achados, vale ressaltar que o Sistema de Tutoria Institucional (SIT) não possui indicadores suficientes para acompanhar a trajetória escolar eficiente e que $42,11 \%$ dos tutores realizam manualmente um acompanhamento individual de acordo com suas necessidades de experiência e informação. A partir deste estudo, foram identificados os indicadores de trajetória escolar que os tutores necessitam para dar um acompanhamento bem-sucedido.

Palavras-chave: controle do desempenho escolar, abandono escolar, reprovação escolar, indicadores educacionais, atraso escolar, monitoramento escolar, trajetória acadêmica, tutoria.

Fecha Recepción: Octubre 2019

Fecha Aceptación: Enero 2020

\section{Introducción}

Según Romo (2011), la tutoría debe proveer una atención individual y personalizada a los estudiantes del nivel superior, con el propósito de que alcancen pleno desarrollo teniendo en cuenta su situación académica y social. Las instituciones educativas deben ofrecer, mediante este tipo de procesos, servicios educativos que atiendan aspectos relacionados con el rezago, la reprobación y la deserción escolar (Domingo, Fernández y Barrero, 2016; Vera et al., 2012). Por lo que la Universidad Autónoma del Carmen (Unacar), a través de la Dirección General de Servicios al Estudiante, ha implementado el Programa Institucional de Tutorías (PIT) con la participación de 236 tutores (profesores de tiempo completo).

La Unacar (2018), en su Modelo Educativo Acalán, ha definido la tutoría como el acompañamiento, orientación, asesoría, canalización y seguimiento que recibe el estudiante por parte de uno o varios tutores a lo largo de su trayectoria escolar para que optimice aprendizajes significativos, potencialice talentos, solucione dificultades personales y escolares y desarrolle hábitos de trabajo y estudio efectivos, todo lo cual le permita ser competente en el área en la que 


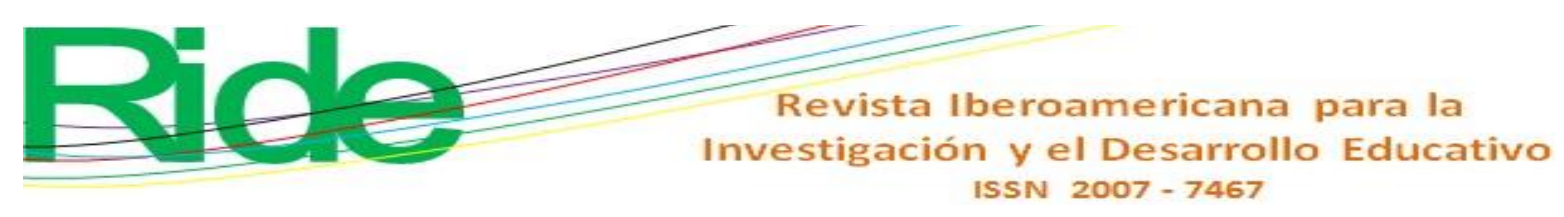

se desarrolle. El trabajo de tutoría exige acompañar al alumno desde su admisión hasta su egreso y titulación, ya que la calidad de los programas educativos está en función del desempeño escolar y el tiempo en que los estudiantes concluyen su preparación profesional. Sin embargo, al dar un servicio individual y personalizado a estos, es importante considerar las características, condiciones y expectativas que tienen al realizar su trayectoria académica (Unacar, 2018).

El tutor o tutora es un profesor de tiempo completo con experiencia en docencia e investigación que atiende a un grupo de estudiantes mediante el análisis de su desempeño académico y los orienta para su desarrollo integral (Unacar, 2002).

Es responsabilidad de los tutores la identificación oportuna de alumnos en riesgo de abandono de estudios, rezago, reprobación o con obstáculos de aprendizaje, así como la intervención temprana para la solución de estos problemas (Alvarado, Vega, Cepeda y Del Bosque, 2014; Mendivil y Ponce, 2016; Romo, 2011).

En el PIT de la Unacar participan los profesores de tiempo completo adscritos a cada una de las escuelas o facultades. Dichos profesores adoptan el rol de tutor o guía académico de un grupo de estudiantes. Desde el momento en que ingresan a la institución a cada estudiante se le asigna uno de estos, quienes tienen la tarea, entre otras, de dar seguimiento a su trayectoria escolar.

La Unacar, ubicada en el sureste de México, ha dispuesto una aplicación web llamada Sistema Institucional de Tutorías [SIT] (Coordinación General de Tecnologías de la Información y la Comunicación de la Unacar [Cgtic], 2014) para el acceso a información académica y personal de los estudiantes que tiene asignados el tutor. El SIT permite el registro del plan de acción tutorial, la identificación de tutorados, el registro de sesiones individuales y grupales con los tutorados, las canalizaciones a otros servicios estudiantiles o de apoyo y consultar el historial académico, entre otras cosas.

La tutoría se ha enfocado por años a la orientación personal y académica, a la socialización y canalización de los estudiantes (Unacar, 2002); sin embargo, el seguimiento ha sido uno de los retos más importantes de la tutoría debido a que la información se encuentra segmentada en los sistemas institucionales. El SIT no integra toda la información y muchos datos que pueden considerarse relevantes no se encuentran aún disponibles en medios digitales.

Actualmente, la institución no proporciona una plataforma de información que aporte datos suficientes para detectar el rezago, los problemas de rendimiento escolar o alumnos en riesgo de deserción, por lo que el trabajo de tutoría se complica. Esta situación ha llevado a la institución a 


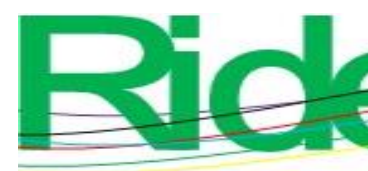

Revista Iberoamericana para la Investigación y el Desarrollo Educativo ISSN $2007-7467$

plantearse la necesidad de aplicar una reingeniería a los sistemas institucionales para poder mejorar el acceso a la información. Y para ello es de suma importancia de reunir la opinión y necesidades de los tutores. Consecuentemente, se planteó un estudio que involucrara a los tutores de la Unacar con el propósito de identificar las necesidades de información para dar un mejor seguimiento de las trayectorias académicas de sus estudiantes. ¿El punto de partida? Determinar cuáles son los indicadores educativos que se requieren procesar.

\section{Método}

Se realizó una investigación transeccional cuantitativa con alcance descriptivo con el fin de precisar los métodos o técnicas que están utilizando los tutores para el seguimiento de las trayectorias escolares de sus estudiantes, eso por una parte; y por la otra, con el objetivo de determinar la lista de indicadores o datos que requieren para mejorar este proceso (Hernández, Fernández y Baptista, 2014).

Para este estudio se diseñó una encuesta de ocho reactivos estructurados en dos secciones. La primera permite reunir información sobre la experiencia tutorial de los encuestados; y la segunda parte integra preguntas que reúnen información sobre los indicadores o datos que se requieren para dar un seguimiento formal de los estudiantes. La recolección de la información se hizo mediante una aplicación web y la invitación fue enviada a todos los tutores vía correo electrónico, emitido por la Coordinación de Tutoría de la Dirección de Servicios al Estudiante de la Unacar.

La población de tutores que colaboran en el PIT son un total de 236 profesores adscritos a las ocho facultades (dependencias de educación superior) de la Unacar. La selección de la muestra fue de tipo probabilístico simple, con la participación voluntaria de 114 tutores (véase tabla 1 y 2). Para determinar si el número de participantes era una muestra representativa de la población objetivo con el grado de confianza adecuado para la viabilidad del estudio se obtuvo el coeficiente de alfa de Cronbach (Tabla 3). Cabe señalar que ninguno de los elementos fue eliminado porque ninguno impacta considerablemente en el ya mencionado coeficiente (Tabla 4) (Quero, 2010). El mayor porcentaje de participación se obtuvo de la Facultad de Ciencias de la Salud con 30 tutores, seguido por la Facultad de Ciencias Educativas y la Facultad de Química con 19 participantes cada una (Figura 1). 


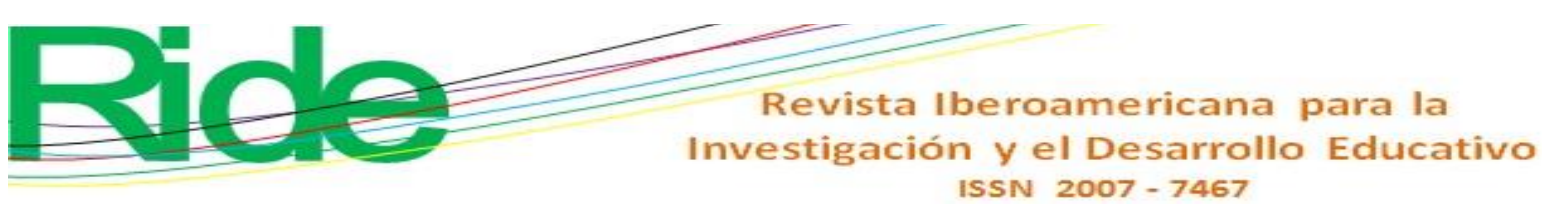

Tabla 1. Población participante en el estudio

\begin{tabular}{|l|c|c|}
\hline \multicolumn{1}{|c|}{$\begin{array}{c}\text { Facultad } \\
\text { (dependencia de educación superior) }\end{array}$} & Tutores & Participantes \\
\hline Derecho & 8 & 6 \\
\hline Ciencias Naturales & 12 & 10 \\
\hline Ciencias Educativas & 26 & 19 \\
\hline Ciencias de la Información & 22 & 9 \\
\hline Ciencias Económicas Administrativas & 40 & 3 \\
\hline Ingeniería & 54 & 12 \\
\hline Química & 26 & 19 \\
\hline Ciencias de la Salud & 48 & 30 \\
\hline No indicaron & - & 6 \\
\hline Total & 236 & 114 \\
\hline
\end{tabular}

Fuente: Elaboración propia

Tabla 2. Resumen de procesamientos de casos

\begin{tabular}{|l|l|c|c|}
\hline \multicolumn{2}{|c|}{} & N & \% \\
\hline Casos & Válido & 114 & 100.0 \\
\cline { 2 - 4 } & Excluido & 0 & 0.0 \\
\cline { 2 - 4 } & Total & 114 & 100.0 \\
\hline
\end{tabular}

Fuente: Elaboración propia

Tabla 3. Estadísticas de fiabilidad

\begin{tabular}{|c|c|}
\hline Alfa de Cronbach & Núm. de elementos \\
\hline 0.951 & 6 \\
\hline
\end{tabular}

Fuente: Elaboración propia 
Tabla 4. Estadística del total de elementos

\begin{tabular}{|l|c|c|c|c|}
\hline \multicolumn{1}{|c|}{ Elementos } & $\begin{array}{c}\text { Media de } \\
\text { escala si el } \\
\text { elemento se } \\
\text { ha suprimido }\end{array}$ & $\begin{array}{c}\text { Varianza de } \\
\text { escala si el } \\
\text { elemento se ha } \\
\text { suprimido }\end{array}$ & $\begin{array}{c}\text { Correlación } \\
\text { total de } \\
\text { elementos } \\
\text { corregida }\end{array}$ & $\begin{array}{c}\text { Alfa de } \\
\text { Cronbach } \\
\text { si el } \\
\text { elemento } \\
\text { se ha } \\
\text { suprimido }\end{array}$ \\
\hline Experiencia en el PIT & 15.24 & 24.395 & .868 & .939 \\
\hline $\begin{array}{l}\text { Participación en el PIT } \\
\text { constante y dinámica }\end{array}$ & 15.27 & 24.766 & .874 & .938 \\
\hline $\begin{array}{l}\text { Atención al seguimiento de } \\
\text { trayectorias escolares }\end{array}$ & 15.29 & 24.579 & .824 & .944 \\
\hline $\begin{array}{l}\text { Acceso a la información en el } \\
\text { SIT }\end{array}$ & 15.32 & 25.156 & .789 & .948 \\
\hline $\begin{array}{l}\text { Identificación del estatus de los } \\
\text { tutorados }\end{array}$ & 15.29 & 24.632 & .819 & .945 \\
\hline Indicadores para el seguimiento & 15.18 & 24.925 & .923 & .934 \\
\hline
\end{tabular}

Fuente: Elaboración propia

Figura 1. Participación de tutores por facultad

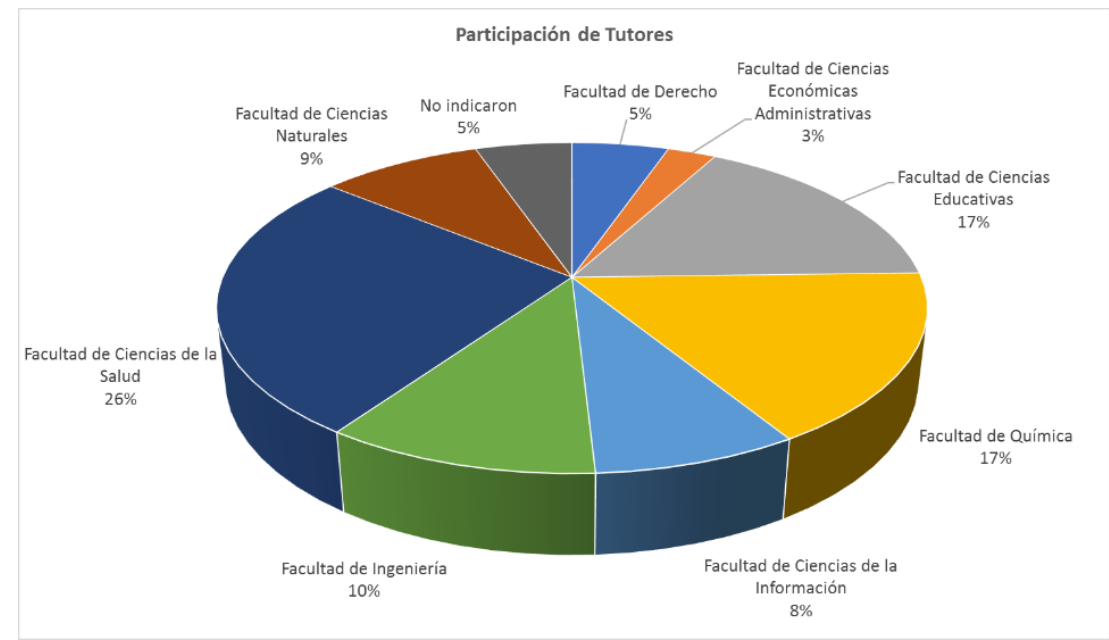

Fuente: Elaboración propia 


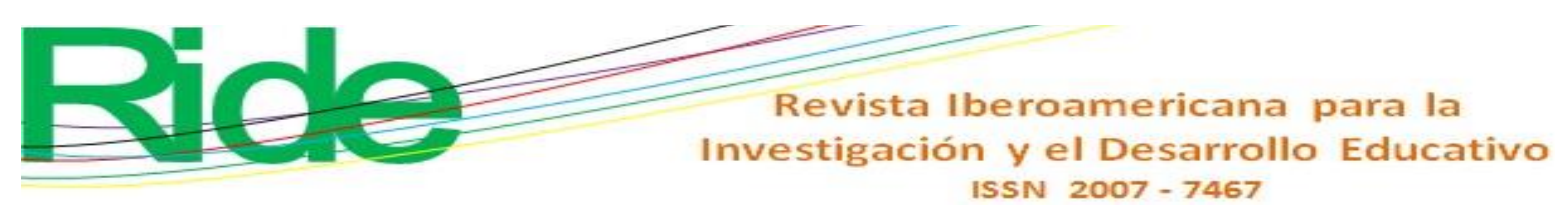

\section{Resultados}

Para dar validez y confianza a la opinión emitida por los profesores se indagó en la experiencia que cada uno tiene como tutor con cuatro preguntas. La primera fue con referencia al número de años que ha participado como tutor en el PIT; la segunda estuvo relacionada con el promedio de tutorados que atiende cada uno de los encuestados en cada ciclo escolar. La tercera pregunta reúne información sobre la técnica o estrategia que utilizan los tutores para seguir la trayectoria escolar de los estudiantes, y con la última pregunta de esta sección se reunió la opinión de los usuarios con respecto al SIT en el seguimiento de las trayectorias escolares.

Figura 2. Experiencia de los profesores expresada en años

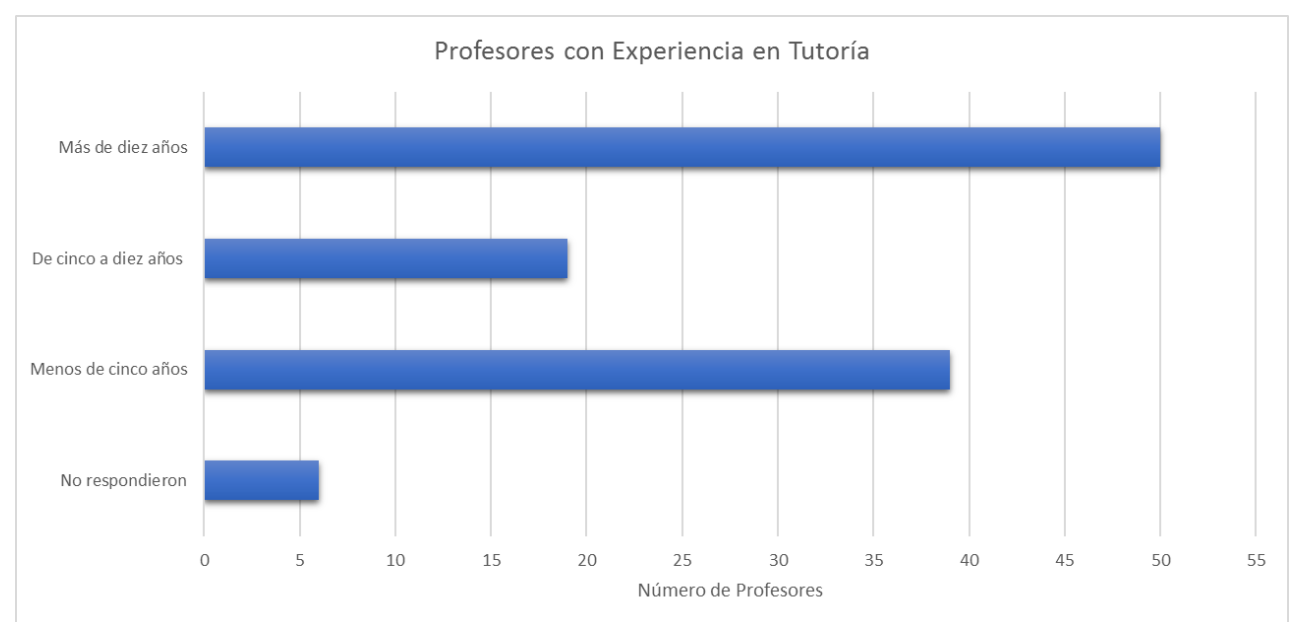

Fuente: Elaboración propia

Con relación al tiempo de experiencia que tienen los profesores en la participación del PIT, se obtuvo que de los 114 tutores que participaron en la encuesta, $43.86 \%$ de ellos tiene una experiencia que supera los 10 años de tutor y $16.67 \%$ ha sido tutor entre 5 y 10 años (Figura 2).

El promedio de tutorados atendidos por cada docente es un factor importante en esta labor, no solo porque a mayor número de tutorados mayor experiencia, sino también debido a que se requiere de técnicas sistematizadas para poder atender, guiar, apoyar y dar seguimiento a un gran número de estudiantes en el mismo tiempo asignado para realizar la tutoría. De las encuestas se obtuvo que $82 \%$ atiende más de 11 tutorados por ciclo escolar, y que de estos $43 \%$ supera los 20 estudiantes (Figura 3). 


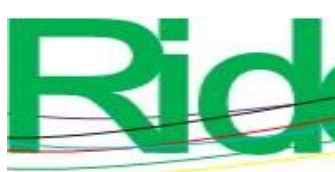

Por otra parte, la Unacar ofrece el servicio de una aplicación web, la ya mencionada SIT, que entre otras funciones permite ver el historial académico del estudiante, el registro de sesiones y la realización de canalizaciones; sin embargo, se cuestionó el uso de la aplicación para el seguimiento de las trayectorias escolares dando a elegir a los profesores encuestados entre tres posibles respuestas: el uso de una técnica de seguimiento propia del tutor, el uso del SIT y la alternativa de que no realiza un seguimiento formal, sino que la tutoría se limita a la atención y canalización (Figura 4). Más de $42 \%$ de los profesores respondió que realiza un seguimiento de acuerdo con su experiencia y necesidades, de forma independiente al SIT. Por otra parte, $38.60 \%$ asegura que utiliza el SIT para realizar la labor tutorial; mientras que $14.04 \%$ no realiza actividades de seguimiento.

Figura 3. Relación de alumnos asignados a cada tutor en el PIT

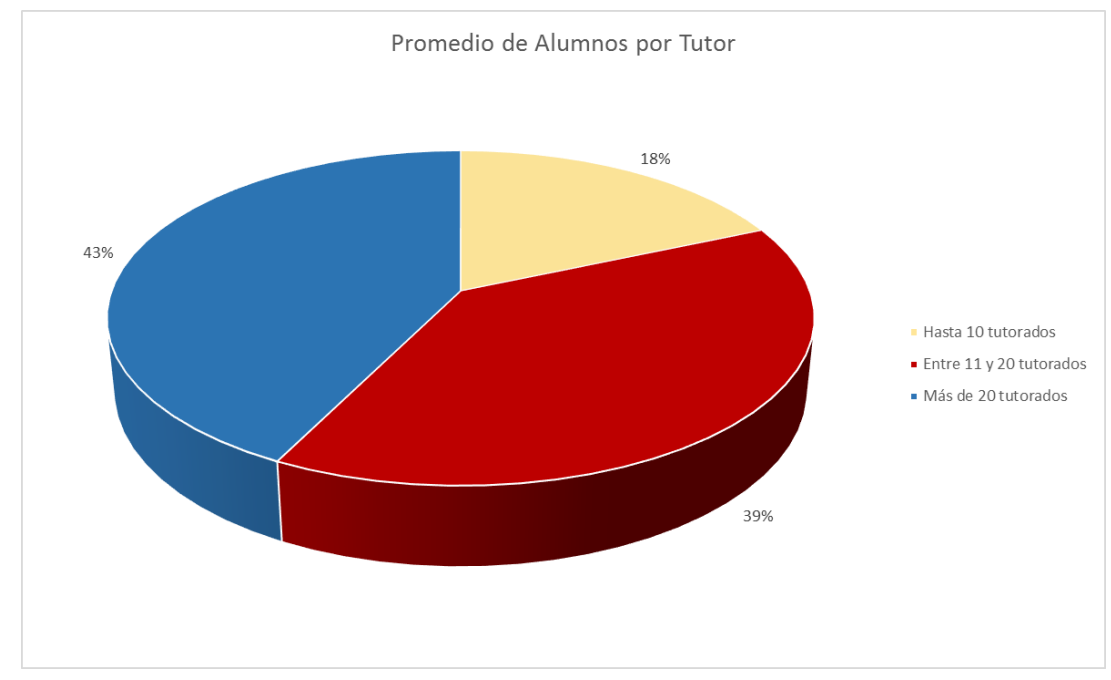

Fuente: Elaboración propia 

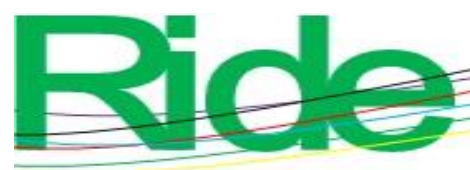

Revista Iberoamericana para la Investigación y el Desarrollo Educativo ISSN $2007-7467$

Figura 4. Método para el seguimiento de trayectorias escolares

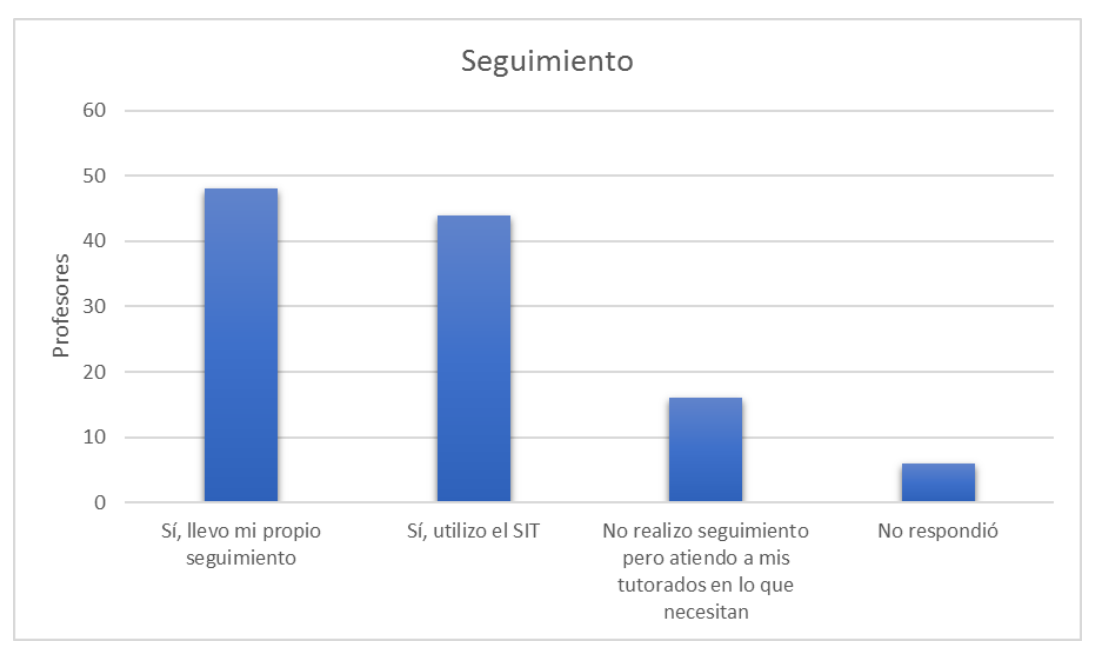

Fuente: Elaboración propia

Por último, se cuestionó a los tutores con respecto a la utilidad de la información presentada por el SIT (fFigura 5). De esto se obtuvo que $37 \%$ está satisfecho con la información que puede consultar en el SIT; sin embargo, 63 \% restante señala que requiere de procesar la información mediante otras herramientas, tales como hojas de cálculo, documentos, aplicaciones estadísticas, entre otros.

Figura 5. Acceso a la información de trayectorias escolares desde el SIT

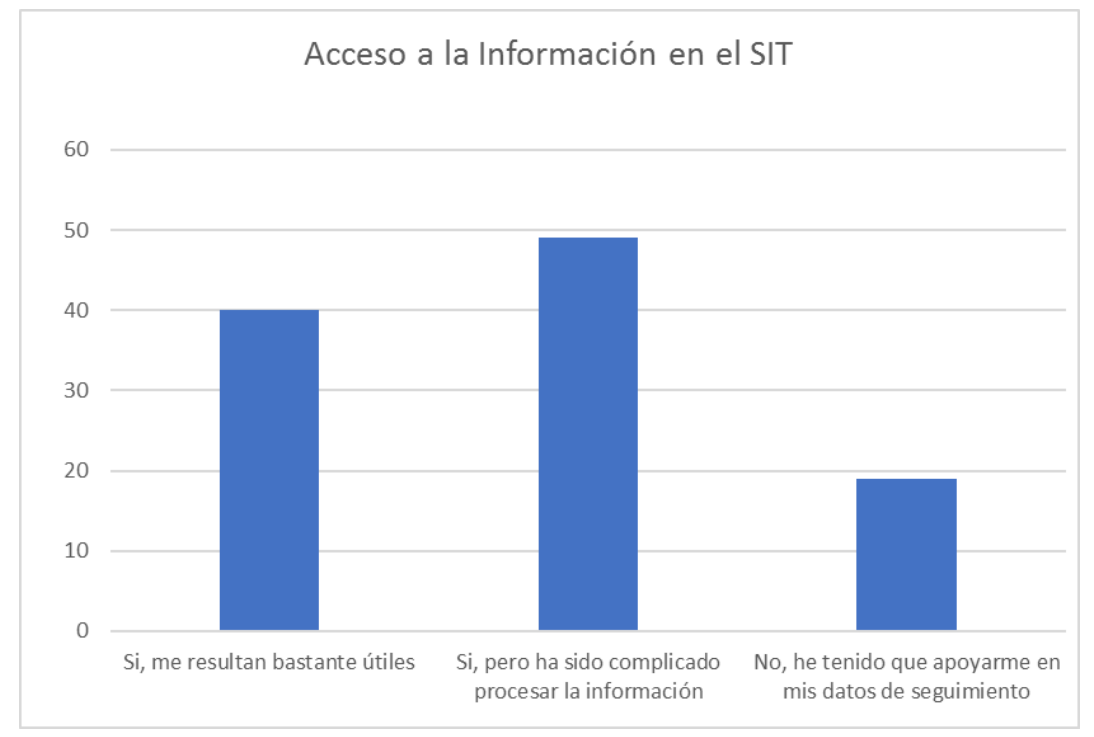

Fuente: Elaboración propia

La segunda sección del instrumento se enfocó en las necesidades de información que se requieren satisfacer para dar un seguimiento formal y confiable de las trayectorias escolares, a 


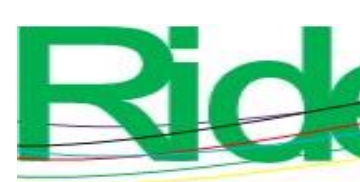

Revista Iberoamericana para la Investigación y el Desarrollo Educativo ISSN 2007 - 7467

saber, según Altamira Rodríguez (citado en Ortega, 2015): los aspectos cuantificables (índices o indicadores) del avance de los estudiantes en sus estudios profesionales, desde el ingreso, durante su permanencia y el egreso.

Se cuestionó a los tutores con respecto a los diferentes términos o indicadores que facilitarían la identificación de los estudiantes de acuerdo con su situación académica actual. Y como parte de ello se propusieron seis estatus que ya existen en otras aplicaciones institucionales, como el Sistema de Control Escolar (SUCE+) y el Portal Ejecutivo, que son sistemas a los que no tienen acceso los tutores (Tabla 5). Los estatus puestos a consideración de los tutores tuvieron una aprobación superior a $45 \%$. Por otra parte, se dio a los tutores la posibilidad de proponer otros indicadores de estatus para un mejor seguimiento de las trayectorias académicas. Los más sugeridos fueron la identificación de alumnos en movilidad, en riesgo de baja definitiva, estudiantes con rezago y los alumnos que están por egresar pero que no han realizado el trámite del certificado profesional (Tabla 6).

Tabla 5. Indicadores de estatus

\begin{tabular}{|l|l|c|}
\hline \multicolumn{1}{|c|}{ Indicador } & \multicolumn{1}{|c|}{ Descripción } & Porcentaje \\
\hline Alumno activo & $\begin{array}{l}\text { Alumno que se encuentra inscrito en el ciclo actual con al } \\
\text { menos un curso en su carga académica. }\end{array}$ & $88 \%$ \\
\hline $\begin{array}{l}\text { Alumno activo } \\
\text { sin cursos }\end{array}$ & $\begin{array}{l}\text { Estudiante que se encuentra activo en el programa educativo } \\
\text { pero que en el ciclo actual no realizó la carga de cursos. }\end{array}$ & $53 \%$ \\
\hline $\begin{array}{l}\text { Alumno en baja } \\
\text { temporal }\end{array}$ & $\begin{array}{l}\text { Estudiante que ha gestionado ante Control Escolar su estatus de } \\
\text { baja temporal o presenta el estatus de activo sin cursos por más } \\
\text { de un periodo escolar. }\end{array}$ & $59 \%$ \\
\hline $\begin{array}{l}\text { Alumno en baja } \\
\text { definitiva }\end{array}$ & $\begin{array}{l}\text { Tutorado que ha gestionado ante la institución su baja definitiva } \\
\text { del programa educativo. }\end{array}$ & $45 \%$ \\
\hline Egresado & Estudiante que ha obtenido su certificado profesional. & $48 \%$ \\
\hline Titulado & Egresado que cuenta con el título profesional. & $48 \%$ \\
\hline Otro & $\begin{array}{l}\text { Los tutores propusieron estatus que no se muestran en ningún } \\
\text { sistema institucional. }\end{array}$ & $22 \%$ \\
\hline
\end{tabular}

Fuente: Elaboración propia 


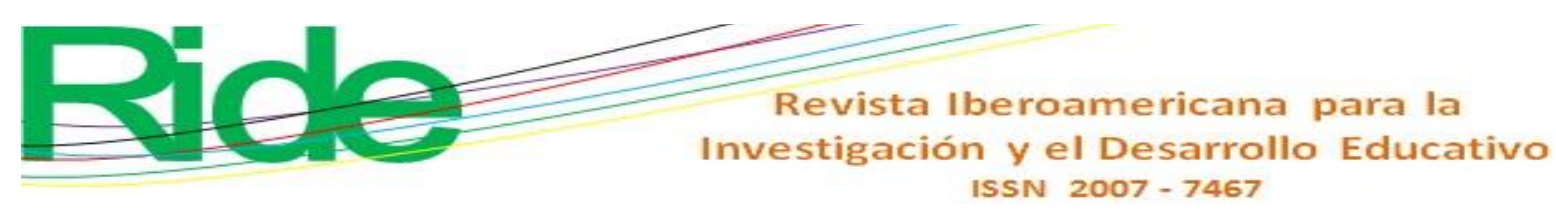

Tabla 6. Otros indicadores de estatus

\begin{tabular}{|l|l|}
\hline \multicolumn{1}{|c|}{ Indicador } & \multicolumn{1}{c|}{ Descripción } \\
\hline Alumno en movilidad & $\begin{array}{l}\text { Tutorado que se encuentra realizando estudios en otra universidad } \\
\text { como parte del programa de movilidad nacional o internacional. }\end{array}$ \\
\hline Alumno en rezago & Alumno con al menos un curso reprobado. \\
\hline Alumno en riesgo de baja & Alumno que ha reprobado el mismo curso al menos dos veces. \\
\hline $\begin{array}{l}\text { Alumno con malla } \\
\text { completa }\end{array}$ & $\begin{array}{l}\text { Alumno que ha completado el total de créditos del plan de } \\
\text { estudios, pero no ha gestionado el certificación profesional. }\end{array}$ \\
\hline
\end{tabular}

Fuente: Elaboración propia

Para el estudio se propuso a los tutores una serie de indicadores (Tabla 7) que consideran la individualidad en el proceso de aprendizaje y el atributo de currículo flexible (flexibilidad en tiempo y elección de cursos) que fundamenta la filosofía del Modelo Educativo Acalán de la Unacar (2018). Es preciso señalar que estos indicadores están disponibles en otros sistemas institucionales a los que no tienen acceso los tutores; están disponibles para el personal del área de Control Escolar y gestores de programas educativos. En la Tabla 7 se puede observar que todos los indicadores que fueron puestos a consideración obtuvieron un nivel de aprobación por encima de $50 \%$. De igual forma, se solicitó a los participantes que propusieran indicadores que estuvieran utilizando en su seguimiento individual y que no fueran considerados en la propuesta, pero no realizaron aportaciones al ser considerados suficientes. 


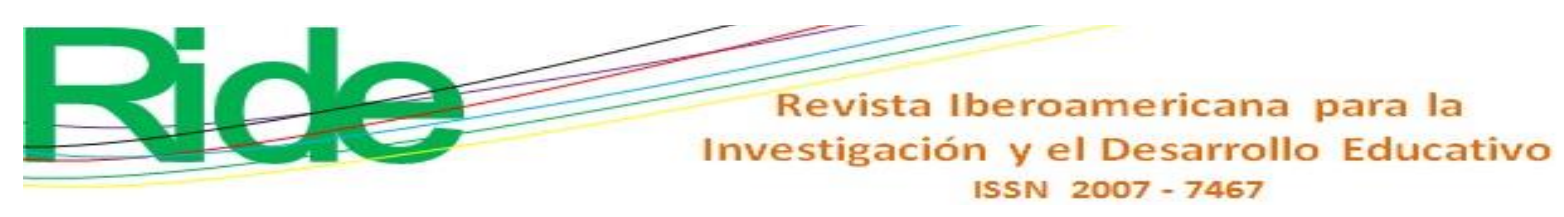

Tabla 7. Indicadores de trayectoria escolar

\begin{tabular}{|c|c|c|}
\hline Indicador & Descripción & Porcentaje \\
\hline Generación & $\begin{array}{l}\text { Periodo escolar de la cohorte generacional al que se integró el } \\
\text { alumno. }\end{array}$ & $74.56 \%$ \\
\hline Ciclos & $\begin{array}{l}\text { Número de ciclos largos (18 semanas) que han transcurrido } \\
\text { desde su ingreso. }\end{array}$ & $73.68 \%$ \\
\hline Cursos totales & $\begin{array}{l}\text { Número de cursos curriculares que integran el programa } \\
\text { educativo. }\end{array}$ & $67.54 \%$ \\
\hline $\begin{array}{l}\text { Cursos } \\
\text { aprobados }\end{array}$ & $\begin{array}{l}\text { Número de cursos que ha aprobado el alumno al momento de } \\
\text { la consulta. }\end{array}$ & $76.32 \%$ \\
\hline Tasa de avance & $\begin{array}{l}\text { Relación }(\%) \text { de cursos aprobados en relación con el total de } \\
\text { cursos del programa educativo. }\end{array}$ & $67.54 \%$ \\
\hline $\begin{array}{l}\text { Tasa de } \\
\text { aprobación }\end{array}$ & Número de cursos aprobados en primera oportunidad. & $65.79 \%$ \\
\hline $\begin{array}{l}\text { Tasa de } \\
\text { promoción }\end{array}$ & $\begin{array}{l}\text { Relación (\%) entre el número de créditos obtenidos con el } \\
\text { total de créditos del programa. }\end{array}$ & $55.26 \%$ \\
\hline $\begin{array}{l}\text { Cursos } \\
\text { reprobados }\end{array}$ & $\begin{array}{l}\text { Número de cursos con calificación reprobatoria que tiene el } \\
\text { alumno al momento de la consulta. }\end{array}$ & $72.81 \%$ \\
\hline Cursos activos & $\begin{array}{l}\text { Número de cursos que está cursando el alumno en el ciclo } \\
\text { actual. }\end{array}$ & $63.16 \%$ \\
\hline $\begin{array}{l}\text { Cursos } \\
\text { pendientes }\end{array}$ & $\begin{array}{l}\text { Número de cursos que el alumno debe acreditar sin considerar } \\
\text { los que está cursando. }\end{array}$ & $67.54 \%$ \\
\hline $\begin{array}{l}\text { Cursos por } \\
\text { acreditar }\end{array}$ & $\begin{array}{l}\text { Número de cursos que no han sido aprobados. Sumatoria de } \\
\text { cursos pendientes con cursos activos. }\end{array}$ & $60.53 \%$ \\
\hline $\begin{array}{l}\text { Actividades de } \\
\text { formación } \\
\text { integral }\end{array}$ & $\begin{array}{l}\text { Número de horas de actividades de formación integral hasta el } \\
\text { momento de la consulta. }\end{array}$ & $69.30 \%$ \\
\hline $\begin{array}{l}\text { Prácticas } \\
\text { profesionales }\end{array}$ & $\begin{array}{l}\text { Estatus del alumno en las prácticas profesionales: acreditado, } \\
\text { no acreditado o en proceso. }\end{array}$ & $66.67 \%$ \\
\hline Servicio social & $\begin{array}{l}\text { Estatus del alumno en el servicio social: acreditado, no } \\
\text { acreditado o en proceso. }\end{array}$ & $66.67 \%$ \\
\hline
\end{tabular}




\begin{tabular}{|c|c|c|}
\hline Movilidad & $\begin{array}{l}\text { Ciclo escolar en el que el alumno fue beneficiario del } \\
\text { programa de movilidad, incluyendo: universidad en donde } \\
\text { estudió, país y beca obtenida. }\end{array}$ & $57.02 \%$ \\
\hline Becas & $\begin{array}{l}\text { Listado de becas con las que se ha beneficiado el alumno en } \\
\text { cada ciclo escolar. }\end{array}$ & $58.77 \%$ \\
\hline $\begin{array}{l}\text { Seguro } \\
\text { facultativo }\end{array}$ & $\begin{array}{l}\text { Número de seguro social. En caso de no tenerlo indicar que no } \\
\text { ha realizado el trámite. }\end{array}$ & $55.26 \%$ \\
\hline $\begin{array}{l}\text { Lista de cursos } \\
\text { por acreditar }\end{array}$ & $\begin{array}{l}\text { Nombre de cada curso. En caso de haberla cursado se incluye } \\
\text { el número de veces que se ha reprobado, indicativo de si lo } \\
\text { está cursando actualmente. }\end{array}$ & $57.89 \%$ \\
\hline $\begin{array}{l}\text { Listado por } \\
\text { ciclo escolar }\end{array}$ & $\begin{array}{l}\text { Tabla generada por ciclo escolar que incluye: ciclo escolar, } \\
\text { estatus del alumno, número de cursos cargados, número de } \\
\text { cursos aprobados, número de cursos reprobados, promedio del } \\
\text { ciclo. }\end{array}$ & $57.89 \%$ \\
\hline Egreso & Periodo escolar y/o fecha en que el alumno egresó. & $56.14 \%$ \\
\hline Titulación & Fecha de titulación y modalidad. & $53.51 \%$ \\
\hline $\begin{array}{l}\text { Rendimiento } \\
\text { escolar }\end{array}$ & $\begin{array}{l}\text { Promedio del alumno considerando la última calificación de } \\
\text { cada curso. }\end{array}$ & $70.18 \%$ \\
\hline Tutor actual & $\begin{array}{l}\text { Nombre del tutor asignado en el ciclo escolar actual. Con la } \\
\text { posibilidad de ver la lista de tutores asignados en ciclos } \\
\text { anteriores. }\end{array}$ & $63.16 \%$ \\
\hline
\end{tabular}

Fuente: Elaboración propia

\section{Discusión}

Un seguimiento eficiente de las trayectorias escolares de los estudiantes depende de contar con la información suficiente, oportuna y confiable para poder realizar detecciones y determinar las estrategias que puedan dar solución a los diversos retos que deben enfrentar los estudiantes e incluso la institución educativa. La meta de los estudiantes es concluir con los estudios profesionales, pero para las instituciones de educación superior es también elevar el índice de eficiencia terminal en sus programas educativos (Rodríguez, 2017; Vásquez, Espino y Olaguez Torres, 2015). 


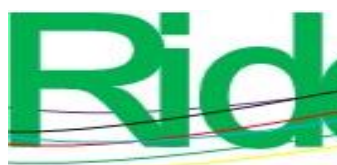

Revista Iberoamericana para la Investigación y el Desarrollo Educativo ISSN $2007-7467$

En la Unacar el seguimiento de las trayectorias escolares es una responsabilidad compartida entre los tutores y los gestores de programas educativos. Sin embargo, el trato individual y personal se da entre los tutores y los estudiantes que tienen asignados. De acuerdo con el estudio, $43 \%$ de los tutores tiene asignado más de 20 alumnos a los que debe dar seguimiento y apoyo, por lo que es importante integrar y procesar la información de cada tutorado para permitir una labor de acompañamiento efectivo y oportuno.

La eficaz selección de los indicadores determinará el éxito del PIT, puesto que con la consulta del expediente del tutorado se podrán identificar los posibles riesgos de rezago o deserción. Indicadores como la tasa de avance, cursos reprobados y el número de cursos por acreditar permitirá a los tutores establecer estrategias para la regularización de los alumnos; indicadores como la tasa de promoción y el rendimiento escolar favorecerá la identificación y el reconocimiento de los alumnos sobresalientes (Guevara y Belelli, 2013).

Los indicadores propuestos en este estudio tienen una relación directa con las tres dimensiones propuestas por Chain y Ramírez (1997) (Figura 6): la dimensión de tiempo, esto es, la continuidad o discontinuidad de los estudios del alumno en relación con su generación, la dimensión de eficiencia escolar, que se refiere a la forma en que los estudiantes aprueban los cursos, y la dimensión de rendimiento escolar, que representa el promedio o resultados del alumno en los diferentes cursos a los cuales se ha inscrito. 


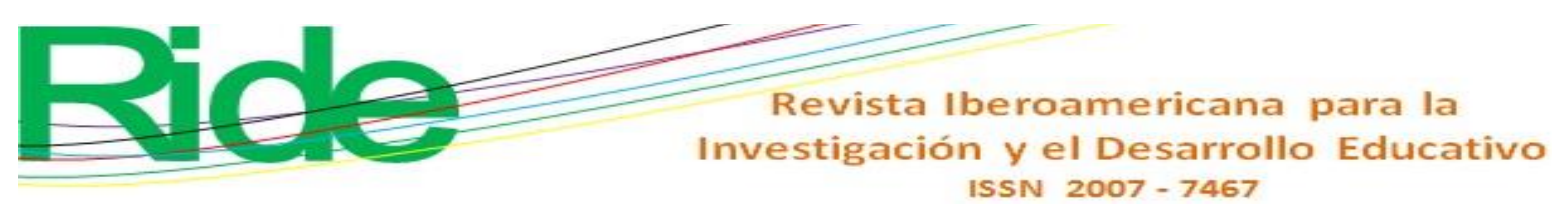

Figura 6. Clasificación de indicadores

\begin{tabular}{l}
\hline Dimensión Tiempo \\
- Generación \\
- Ciclos \\
\hline - Cursos Aprobados \\
- Tasa de Aprobación \\
- Cursos Reprobados \\
- Cursos Activos \\
- Cursos Pendientes \\
- Cursos por Acreditar \\
- Lista de Cursos por Acreditar \\
- Listado por Ciclo Escolar \\
\hline Dimensión Rendimiento Escolar \\
- Tasa de Avance \\
- Tasa de Promoción \\
- Egreso \\
- Titulación
\end{tabular}

Fuente: Elaboración propia con base en Chain y Ramírez (1997)

Por otra parte, se han propuesto indicadores que no corresponden a ninguna de las dimensiones establecidas por dicha dupla de autores pero que por la flexibilidad del modelo educativo de la institución resulta importante consultar, entre ellos las actividades de formación integral, prácticas profesionales, servicio social, el programa de movilidad, becas y el seguro médico facultativo.

El estudio involucró a los tutores de la institución con una participación voluntaria de $48 \%$, quienes contribuyeron con su experiencia en el PIT y brindaron propuestas útiles que apoyarán a la mejora del seguimiento de trayectorias escolares. Por otra parte, ha sido de gran valor el descubrir que $45 \%$ de los tutores debe procesar con otras herramientas la información que proporciona el SIT; por consiguiente, se debe considerar un proceso de reingeniería en la aplicación para que permita el acceso a información que facilite el trabajo tutorial. 


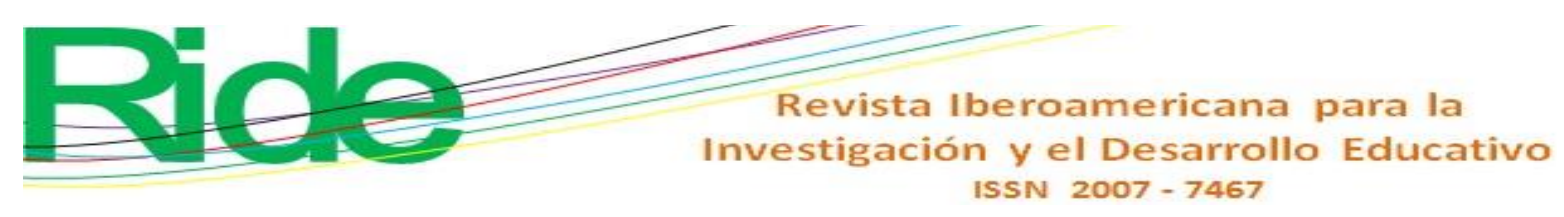

\section{Conclusiones}

La Unacar pasó de tener un modelo educativo tradicional a un modelo educativo centrado en el aprendizaje con enfoque en competencias, lo que dio flexibilidad a los programas educativos de nivel superior y permitió que los estudiantes elijan los cursos y el tiempo que pueden tardar en concluir los estudios profesionales. Sin embargo, aún es un reto definir las estrategias que permitan a las instituciones de educación superior en general superar los altos índices de deserción, reprobación y rezago, al igual que los bajos índices de eficiencia terminal.

La tutoría académica es el medio para identificar de manera oportuna los problemas académicos que presentan los estudiantes y que afectan directamente a la eficiencia terminal de los programas educativos; por lo tanto, la reestructuración del seguimiento de las trayectorias escolares se inició con la evaluación desde la perspectiva del tutor, con la identificación y aprobación de los indicadores de trayectoria académica individual, lo cual permitirá arrancar un proceso de reingeniería en la evaluación y en el seguimiento que se realizan en el PIT a través del SIT.

Con este estudio se propusieron una serie de indicadores que permitirán al tutor dar seguimiento al estatus actual del estudiante, los logros y áreas de oportunidad para lograr el egreso de acuerdo con su cohorte generacional.

\section{Futuras líneas de investigación}

Como futura líneas de investigación se ha propuesto evaluar el acceso y necesidades de información de las gestorías de los programas educativos de nivel superior para que, aunado a la determinación de una serie de indicadores y la forma de medirlos o calcularlos, se pueda dar solución al seguimiento de trayectorias escolares y el acceso a la información. Además, se podría identificar y combatir otros problemas académicos como la deserción, rezago y eficiencia terminal.

\section{Agradecimientos}

Se agradece a la Dirección General de Investigación y Posgrado el apoyo recibido a través del proyecto CAIPI/2018/01 y a la Dirección General de Servicios al Estudiante de la institución objeto de estudio por su importante colaboración. De igual manera, se agradece la colaboración de la Ing. Rocío Guadalupe Laines López por su valiosa colaboración en el desarrollo del proyecto. 


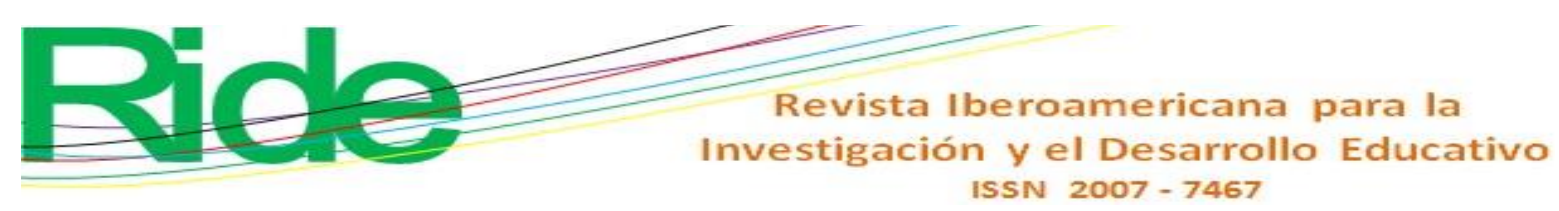

\section{Referencias}

Alvarado, I. R., Vega, Z., Cepeda, M. L. y Del Bosque, A. E. (2014). Comparación de estrategias de estudio y autorregulación en universitarios. Revista Electrónica de Investigación Educativa, 16(1), 137-148.

Chain, R. y Ramírez, C. (1997). Trayectoria escolar: La eficiencia terminal en la Universidad Veracruzana. Revista de la Educación Superior, 26(102), 79-97.

Coordinación General de Tecnologías de la Información y la Comunicación de la Universidad Autónoma del Carmen [Cgtic]. (2014). Sistema Institucional de Tutoría (SIT) (versión 2.0). México: Universidad Autónoma del Carmen. Recuperado de http://charal.unacar.mx/sitweb2.0/login.aspx.

Domingo, J., Fernández, J. D. y Barrero, B. (2016). El orientador ante el reto de la mejora curricular. Un estudio de caso. Revista Electrónica de Investigación Educativa, 18(2), 27 39.

Guevara, H. M. y Belelli, S. E. (2013). Las trayectorias académicas: dimensiones personales de una trayectoria estudiantil. Testimonio de un actor. RevIISE - Revista de Ciencias Sociales y Humanas, 4(4), 45-56.

Hernández, R., Fernández, C. y Baptista, P. (2014). Metodología de la Investigación (6. ${ }^{a}$ ed.). México: McGraw-Hill.

Mendivil, G. y Ponce, S. (2016). La trayectoria escolar en la formación inicial de profesores de matemáticas. RIDE Revista Iberoamericana para la Investigación y el Desarrollo Educativo, 7(13), 370-387.

Ortega, J. C. (2015). Surgimiento de la propuesta del estudio de las Trayectorias Escolares en la Universidad Veracruzana. En Ortega, J. C., López, R. y Alarcón, E. (coords.), Trayectorias escolares en educación superior. Propuesta metodológica y experiencias en México (pp. 23-42). Xalapa, México: Universidad Veracruzana.

Quero, M. (2010). Confiabilidad y coeficiente Alpha de Cronbach. TELOS. Revista de Estudios Interdisciplinarios en Ciencias Sociales, 12(2), 248-252.

Rodríguez, I. (2017). La calidad de la educación superior y la reestructuración del programa de tutoría. Revista Iberoamericana para la Investigación y el Desarrollo Educativo, 8(15), 135-154. Recuperado de https://doi.org/10.23913/ride.v8i15.294.

Romo, A. (2011). La tutoría. Una estrategia innovadora en el marco de los programas de atención 


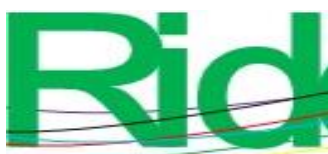

a estudiantes. Ciudad de México, México: Asociación Nacional de Universidades e Instituciones de Educación Superior, Dirección de Medios Editoriales. Recuperado de http://publicaciones.anuies.mx/pdfs/libros/Libro225.pdf.

Universidad Autónoma del Carmen [Unacar]. (2002). Tutoría institucional. Recuperado de http://www.unacar.mx/SERVICIOS_ESTUDANTILES/tutoria/tutoria.html.

Universidad Autónoma del Carmen [Unacar]. (2018). Universidad Autónoma del Carmen. Modelo

Educativo Acalán. Campeche, México: Recuperado de http://www.unacar.mx/Dir_General_Academica/Documentos/Modelo_Educativo_Acalan/ Modelo_Ed_Acalan2017.pdf.

Vásquez, C. R., Espino, P. y Olaguez, J. E. (2015). Repercusiones de la tutoría académica en estudiantes de ingeniería. Revista Iberoamericana para la Investigación y el Desarrollo Educativo, 5(10).

Vera, J. Á., Ramos, D. Y., Sotelo, M. A., Echeverría, S., Serrano, D. M. y Vales, J. J. (2012). Factores asociados al rezago en estudiantes de una institución de educación superior en México. Revista Iberoamericana de Educación Superior, 3(7), 41-56. 


\begin{tabular}{|l|l|}
\hline \multicolumn{1}{|c|}{ Rol de Contribución } & \multicolumn{1}{c|}{ Autor (es) } \\
\hline Conceptualización & Fernando Enrique Sánchez Martínez \\
\hline Metodología & Fernando Enrique Sánchez Martínez \\
\hline Software & NO APLICA \\
\hline Validación & Ricardo Armando Barrera Cámara \\
\hline Análisis Formal & Ana Alberta Canepa Sáenz \\
\hline Investigación & Ana Alberta Canepa Sáenz \\
\hline Recursos & Ricardo Armando Barrera Cámara \\
\hline Curación de datos & Ana Alberta Canepa Sáenz \\
\hline $\begin{array}{l}\text { Escritura - Preparación del } \\
\text { borrador original }\end{array}$ & Fernando Enrique Sánchez Martínez \\
\hline Escritura - Revisión y edición & Ricardo Armando Barrera Cámara \\
\hline Visualización & Fernando Enrique Sánchez Martínez \\
\hline Supervisión & Ricardo Armando Barrera Cámara \\
\hline Administración de Proyectos & Fernando Enrique Sánchez Martínez \\
\hline Adquisición de fondos & Fernando Enrique Sánchez Martínez \\
\hline
\end{tabular}

